\title{
Prevalence of metabolic syndrome among premenopausal and postmenopausal working women in Hyderabad, Pakistan
}

\author{
Aisha Memon ${ }^{1}$, Nimra Masood Baig ${ }^{1}$, Ayaz Ali Samo ${ }^{1 *}$, Khalida Shaikh ${ }^{1}$ \\ and Zulfiqar Ali Laghari ${ }^{1}$ \\ 1. Department of Physiology and Medical Laboratory Technology, University of Sindh, Jamshoro-Pakistan \\ *Corresponding author's email: ayazsamo@usindh.edu.pk \\ Citation \\ Aisha Memon, Nimra Masood Baig, Ayaz Ali Samo, Khalida Shaikh and Zulfiqar Ali Laghari. Prevalence of \\ metabolic syndrome among premenopausal and postmenopausal working women in Hyderabad, Pakistan. Pure and \\ Applied Biology. Vol. 10, Issue 1, pp19-25. http://dx.doi.org/10.19045/bspab.2021.100003
}

\begin{tabular}{llll}
\hline \hline Received: 08/05/2020 & Revised: 05/08/2020 & Accepted: 12/08/2020 & Online First: 26/08/2020 \\
\hline
\end{tabular}

\section{Abstract}

Metabolic Syndrome (MetS) might cause diabetes and cardiovascular diseases. No extensive study regarding prevalence of MetS has been carried out in working women of Hyderabad. The main purpose of this study is to assess the prevalence of MetS among premenopausal and postmenopausal working women. This cross sectional study was carried out from January 2018 to June 2018, on 276 working women, selected from Girls Colleges of public sector in Hyderabad region. The data was collected through structured questionnaire. Venous blood sample was collected while the participants were fasting, and serum was collected after centrifuging the samples. Estimation of triglycerides (TG), cholesterol, low-density lipoprotein (LDL) cholesterol, and high-density lipoprotein (HDL) cholesterol was carried out using Spectra XL fully automatic machine. Systolic blood pressure (SBP) and diastolic blood pressure (DBP) were measured using sphygmomanometer. Out of 276, overall the prevalence of Metabolic Syndrome (MetS) was 46.37 $\%$ in both premenopausal and postmenopausal women. The prevalence of MetS was higher in postmenopausal women $59.06 \%$ than in premenopausal women $31.50 \%$. The most frequent risk component of MetS in premenopausal women was central obesity $72.44 \%$, followed by low HDL cholesterol $68.50 \%$, high triglycerides $31.49 \%$, hypertension $15.74 \%$ and increased fasting blood sugar FBS $7.87 \%$. Whereas, in postmenopausal women the most frequent component was central obesity $71.14 \%$, followed by low HDL cholesterol 59.06\%, hypertension 59.06\%, high triglycerides $38.25 \%$ and increased FBS $12.75 \%$. Prevalence of MetS and the major risk components was higher in postmenopausal women. Postmenopausal women are at higher risk of developing diabetes and cardiac diseases.

Keywords: Dyslipidemia; Menopause; Metabolic Syndrome; Obesity

\section{Introduction}

Metabolic Syndrome is the problem of a concern not only in the developed countries but also in developing countries [1]. MetS is defined as a clustering of cardiovascular risk factors, MetS is also precursor of diabetes and cardiac diseases [2,3]. It is estimated that an individual suffering from the MetS has more likely chances of suffering from cardiac diseases [2]. MetS represents the clustering 
of risk factors, which reflect over-nutrition and sedentary life styles often resulting excessive adiposity [3]. The MetS includes the central abdominal obesity as the major risk component [3], the other risk components are dyslipidemia often characterized by either low HDL cholesterol or increased triglycerides, insulin resistance or increased FBS and elevated systolic and diastolic blood pressure along with other comorbidities and fatty liver disease [4-6].

Increased urbanization has changed the lifestyle during the last century, these changes are sedentary or inactive life style, high calories food, consumption of junk food, all these have been reported as the contributory factors for developing obesity [7]. The MetS affects all age groups after the age of 30 years, however, women having menopause, marked by stoppage of menstrual cycle, are more likely to have higher prevalence of MetS [8, 9]. Higher prevalence of MetS in premenopausal women is alarming, since MetS is also precursor of diabetes and cardiac diseases [10]. In addition to causing the diabetes and cardiac diseases, the participants, who have MetS might also increase risk of deaths due to associated morbidities [11].

It has been reported in previous studies that menopause in women is one of the contributing factors in the prevalence of MetS [8, 9]. After menopause and due to hormonal changes, postmenopausal women are at high risk of cardiovascular diseases as compared to premenopausal women and men in a same age group [12]. The perimenopause is the stage before menopause, which is characterized the irregularity of menstrual cycle changes due to hormonal variability, the women having perimenopause should be aware of MetS, which might be the forthcoming event [13]. In menopausal women, there is deficiency of estrogen hormone, which appears to be the associate factor to clinical features of metabolic syndrome [13]. This has also been reported that postmenopausal women are at more risk of suffering from CVDs, and diabetes [14].

With an increase in the prevalence of MetS worldwide, and in the face of sparse data available in our part of the world, this study has been designed to assess the prevalence of MetS among premenopausal and postmenopausal working women in the selected colleges of Hyderabad, Sindh, Pakistan. Working women mostly represent the sedentary life, which might lead to central obesity; the central obesity is the major risk component of MetS. Since MetS is a diagnostic criterion for diabetes and cardiac diseases, assessing MetS in working women will be helpful in preventive strategies from both diabetes and cardiac diseases.

\section{Materials and Methods}

Study setting: This cross sectional study was carried out from January 2018- June 2018, on 276 working women, selected from selected degree colleges of Hyderabad, Pakistan. All the participants who were aged between 35 to 60 years of age and were permanent residence of Hyderabad were included in the study. All those who had any gynecological disorders, cardiac diseases or any other illness were excluded from the study. Those, who were on medication, were also excluded from the study.

\section{Data collection}

The data was collected through structured questionnaire. Demographic information was achieved. The MetS was assessed using National Cholesterol Education Program, Adult Treatment 111, This criterion does not require insulin resistance yet it requires the presence of any three components from the five risk components [15], these five risk components are central obesity determined by waist circumference $>88$ centimeters, fasting blood glucose $\geq 110 \mathrm{mg} / \mathrm{dl}, \mathrm{TG} \geq 150$ $\mathrm{mg} / \mathrm{dl}$, HDL cholesterol $<50 \mathrm{mg} / \mathrm{dl}$, Systolic Blood Pressure $\geq 130 \mathrm{mmHg}$ and Diastolic Blood pressure $\geq 85 \mathrm{mmHg}$. 
Venous blood sample was collected in the morning time, while respondents were fasting. Serum was collected after centrifuging the samples; serum was kept at $4^{\circ} \mathrm{C}$. Estimation of triglycerides, total cholesterol, LDL cholesterol, and HDL cholesterol was carried out using Spectra XL fully automatic machine. FBS was measured using glucometer from finger prick drop of the blood. The systolic and diastolic blood pressure was obtained using sphygmomanometer.

\section{Anthropometric measurements}

The anthropometric measurements were carried out using standard protocol. The Weight of the participants was measured using the weighing scale. Calibration was done regularly to avoid obtaining the data with errors; Weight was taken without shoes and in light clothing. Height assessment was measured using a height measuring rod without shoes. Waist circumference was measured using a non-stretchable tape at level of the uppermost edge of the hip bone, while participants were wearing light cloths. The waist circumference was divided with hip circumference for obtaining the waist hip ratio.

\section{Ethical approval}

Study was approved by Institutional Review Board of Department of Physiology, Faculty of Natural Sciences, University of Sindh, Jamshoro. Informed consent was obtained before collection of data. Objectives of the study were explained to the participants of the study and all those who were agreed to participate in study were included in the study.

Statistical analysis: Statistical analysis was carried out using SPSS version 23. The data on prevalence of MetS and risk components was presented in percentages, baseline characteristics between premenopausal women and postmenopausal women were compared using the mean values, standard deviation.

\section{Results}

Total 302 participants were recruited for the study, out of 302 only 276 showed their willingness to participate in the study, these participants also gave consent to draw the blood and collect information on the structured questionnaire. The response rate of the participant was $91.39 \%$. Out of 276 participants, 127 (46.02\%) participants were premenopausal and 149 (53.98\%) were postmenopausal. MetS was diagnosed by using the National Education Treatment Program, Adult Panel Treatment 111, NCEP ATP111 criteria. According to the NCEP ATP111 criteria, Overall prevalence of MetS in both premenopausal and postmenopausal women was $46.37 \%$. Postmenopausal women had higher prevalence of MetS $59.06 \%$ than premenopausal women $31.50 \%$ (Table 1 ).

Table 2 shows the prevalence of each component of MetS in premenopausal and postmenopausal women. Overall the prevalence of abdominal or central obesity was $71.73 \%$, however, the premenopausal women had slightly higher prevalence of central obesity $72.44 \%$ than post-menopausal women $71,14 \%$. The prevalence of increased fasting blood glucose was higher in postmenopausal women $12.75 \%$ than premenopausal women $7.87 \%$. The low HDL cholesterol was higher in premenopausal women $68.50 \%$ than in postmenopausal $59.06 \%$, whereas the postmenopausal had higher prevalence of increased Triglyceride $38.25 \%$ and higher SBP 59.06\% than premenopausal women $31.49 \%$ and $15.74 \%$ respectively (Table 2 ). The mean values of participants are presented along with t-test and $P$-value in table 3 . The mean age of the premenopausal women was $39.81 \pm 3.17$ and the mean age of postmenopausal was 52.79 \pm 3.81 . The anthropometric analysis indicated that no significant difference was found between premenopausal and postmenopausal women regarding waist circumference $(\mathrm{t}=0.45, \mathrm{P}>$ 
$0.05)$ and BMI $(\mathrm{t}=1.66, \mathrm{P}>0.05)$. However, premenopausal women had significantly higher WHR $(\mathrm{t}=2.29, \mathrm{P}<0.05)$ than postmenopausal women. The systolic blood pressure $(\mathrm{t}=9.51, \mathrm{P}<0.0001)$ and diastolic blood pressure $(\mathrm{t}=4.33, \mathrm{P}<0.0001)$ was significantly higher in postmenopausal women. Similarly, the fasting blood sugar was significantly higher $(\mathrm{t}=2.83, \mathrm{P}<0.01)$ in postmenopausal women. The lipid profile analysis indicated the significantly higher concentration of total cholesterol $(\mathrm{t}=9.03$, $\mathrm{P}<0.0001)$, HDL cholesterol $(\mathrm{t}=3.76, \mathrm{P}<$ $0.01)$ and LDL cholesterol $(\mathrm{t}=7.11, \mathrm{P}<$ 0.0001 ) in postmenopausal women than in premenopausal women, however, no significant difference $(\mathrm{P}>0.05)$ was found in the level of triglyceride and VLDL both in premenopausal and postmenopausal women (Table 3).

Table 1. Frequency of MetS in premenopausal and postmenopausal women

\begin{tabular}{|c|c|c|c|c|c|c|}
\hline \multirow{2}{*}{ Participants } & \multicolumn{2}{|c|}{ MetS } & \multicolumn{2}{c|}{ Without MetS } & \multicolumn{2}{c|}{ Total } \\
\cline { 2 - 6 } & $\mathbf{N}$ & $\mathbf{\%}$ & $\mathbf{N}$ & $\mathbf{\%}$ & $\mathbf{N}$ & \% \\
\hline Premenopausal women & 40 & 31.50 & 87 & 68.50 & 127 & 46.02 \\
\hline Postmenopausal women & 88 & 59.06 & 61 & 40.94 & 149 & 53.98 \\
\hline Total participants & 128 & 46.37 & 148 & 53.62 & 276 & 100 \\
\hline
\end{tabular}

Table 2. Distribution of MetS risk components in premenopausal and postmenopausal women

\begin{tabular}{|c|c|c|c|c|}
\hline \multirow{2}{*}{ MetS risk components } & \multicolumn{2}{|c|}{ Premenopausal (n=127) } & \multicolumn{2}{c|}{ Postmenopausal (n=149) } \\
\cline { 2 - 5 } & $\mathbf{N}$ & $\mathbf{\%}$ & $\mathbf{N}$ & \% \\
\hline $\mathrm{WC}>88 \mathrm{~cm}$ & 92 & 72.44 & 106 & 71.14 \\
\hline $\mathrm{FBS} \geq 110 \mathrm{mg} / \mathrm{dl}$ & 10 & 7.87 & 19 & 12.75 \\
\hline $\mathrm{HDL}<50 \mathrm{mg} / \mathrm{dl}$ & 87 & 68.50 & 88 & 59.06 \\
\hline $\mathrm{TG} \geq 150 \mathrm{mg} / \mathrm{dl}$ & 40 & 31.49 & 57 & 38.25 \\
\hline $\mathrm{HTN} \geq 130 / \geq 85 \mathrm{mmHg}$ & 20 & 15.74 & 88 & 59.06 \\
\hline
\end{tabular}

Table 3. Distribution of mean and standard deviation values of characteristics of the participants

\begin{tabular}{|c|c|c|c|c|}
\hline Characteristics & Premenopausal $(\mathbf{n}=127)$ & Postmenopausal $(\mathbf{n}=149)$ & t-test & P-value \\
\hline Age (years) & $39.81 \pm 3.17$ & $52.79 \pm 3.81$ & 30.44 & .0001 \\
\hline WC (inches) & $37.18 \pm 3.65$ & $36.99 \pm 3.29$ & 0.45 & 0.64 \\
\hline WHR & $0.87 \pm 0.06$ & $0.85 \pm 0.081$ & 2.29 & .0224 \\
\hline BMI kg/m ${ }^{2}$ & $24.98 \pm 5.19$ & $25.93 \pm 4.27$ & 1.66 & .096 \\
\hline SBP mmHg & $120.32 \pm 12.10$ & $133.87 \pm 11.52$ & 9.51 & $<0.0001$ \\
\hline DBP mmHg & $78.92 \pm 11.93$ & $85.02 \pm 11.43$ & 4.33 & $<0.0001$ \\
\hline FBS mg/dl & $87.16 \pm 33.23$ & $103.06 \pm 55.15$ & 2.83 & 0.0049 \\
\hline CHOL mg/dl & $176.99 \pm 25.38$ & $207.27 \pm 29.60$ & 9.03 & $<0.0001$ \\
\hline TG mg/dl & $138.38 \pm 56.30$ & $144.14 \pm 60.81$ & 0.81 & 0.41 \\
\hline HDL mg/dl & $38.20 \pm 5.30$ & $40.97 \pm 6.69$ & 3.76 & 0.0002 \\
\hline LDL mg/dl & $111.82 \pm 26.74$ & $134.57 \pm 26.24$ & 7.11 & .0001 \\
\hline VLDL mg/dl & $27.43 \pm 9.57$ & $28.52 \pm 12.18$ & 0.81 & .4150 \\
\hline
\end{tabular}

WC: waist circumference, WHR: waist to hip ratio, BMI: body mass index, SBP: systolic blood pressure, DBP: diastolic blood pressure, FBS: fasting blood glucose, CHOL: cholesterol, TG: Triglyceride, HDL: high density lipoproteins, LDL: low density lipoproteins, VLDL: very low density lipoproteins 


\section{Discussion}

The data, we present here shows the prevalence of MetS at $46.37 \%$, this is lower than previously published study conducted in Pakistan, which showed the prevalence of MetS around 57\% in female [16]. The study conducted on diabetes patients showed the higher prevalence of MetS in both male $71.4 \%$ and in female $95.3 \%$, the higher prevalence of MetS is alarming [17]. Another study conducted on diabetes patients showed the higher prevalence of five risk factor $46.2 \%$ [18], which is higher than our study. The higher prevalence of MetS in these studies suggest is due to the fact that studied subjects were diabetes patients, whereas our study was conducted on normal subjects. MetS has been reported from the beginning of menopausal phase to the postmenopausal phase compared to premenopausal women [19], the similar study also showed the prevalence of Metabolic syndrome is $53 \%$ in premenopausal women and $69 \%$ in postmenopausal women, in our present study, we report here the higher prevalence of MetS in postmenopausal women. Our study shows higher prevalence of MetS in postmenopausal women $59.06 \%$. The prevalence of MetS was higher in our study than previously published reports from Bangladesh and Iran $[12,20]$. This might be due to the reason that we selected college teachers which usually had sedentary life style, and also the study is from urban areas, which has previously shown the higher prevalence of MetS, however our study had lesser prevalence of MetS than in Indian study [21].

This study also presents the prevalence of five major risk complements of MetS. The first major risk component is obesity; the data we present here indicate no significant difference in the prevalence of obesity between pre and postmenopausal women. Both premenopausal and postmenopausal women were at equal risk of suffering from central obesity, the results are alarming since some other studies had also reported the higher prevalence of obesity, which needs an intervention to reduce the obesity [11, 17]. However, according to waist hip ratio premenopausal women had higher prevalence of obesity, which might be due to dietary or other sociodemographic factors, this needs to be investigated in the future. The TG level was higher in our study in postmenopausal women; this is consistent with previously reported studies [21]. Surprisingly, the prevalence of low HDL cholesterol level was higher in premenopausal women, which is not consistent with previously published studies $[20,21]$. The possible interpretation might be due to the reason that our study was on healthy adults, since some other studies are in agreement with our findings [22, 23]. Hyperglycemia is one of the risk components of MetS, the findings in this study indicate that postmenopausal women had higher prevalence of increased fasting glucose than premenopausal women, these results are in agreement with previous studies [19], the presence of high glucose level in metabolic syndrome is one of the component that detects the diabetes mellitus, this is why Mets is often regarded as the diagnostic criteria for detecting the diabetes mellitus. Hypertension was found in this study as the component that was higher in postmenopausal women; the increase prevalence of hypertension is one of the factors that detect the CVDs. This has also been reported the higher prevalence of hypertension in postmenopausal women [3, 14].

Metabolic syndrome is the leading cause of CVD risk factors it increases two fold in postmenopausal women compare to premenopausal women $[24,25]$. Our data also showed an increase in number of metabolic syndrome risk components in postmenopausal women, these risk components are also the leading cause of 
CVD risk factors, so postmenopausal women have higher risk of CVD as compare to premenopausal women. The current study indicates the postmenopausal are at higher risk for suffering from CVD due to increased prevalence of cardiovascular risk factors. Our study also reported the higher prevalence of MetS and associated major risk components in postmenopausal workingwomen, which suggest that postmenopausal women are at higher risk of suffering from MetS than premenopausal women.

\section{Conclusion}

Postmenopausal women had higher prevalence of MetS, additionally postmenopausal women had higher frequency of major risk components of MetS. Higher prevalence of MetS in postmenopausal might cause diabetes and cardiac diseases, in this regard the timely intervention and public health policies are needed to reduce the increasing prevalence of MetS particularly in postmenopausal women Authors' contributions

Study concept and design: A Memon, NM Baig, AA Samo \& K Shaikh, Acquisition of data: ZA Laghari, Analysis and interpretation of data: A Memon \& ZA Laghari, Drafting of the manuscript: AA Samo \& ZA Laghari, Critical revision of the manuscript: ZA Laghari, Statistical analysis: ZA Laghari.

\section{References}

1. Zimmet $\mathrm{P}$, Magliano D, Matsuzawa Y, Alberti G \& Shaw J (2005). The metabolic syndrome: a global public health problem and a new definition. $J$ Athero \& Thromb 12(6): 295-300.

2. Ford ES (2005). Risks for all-cause mortality, cardiovascular disease, and diabetes associated with the metabolic syndrome: a summary of the evidence. Diab Care 28(7): 1769-1778.

3. Després J.-P., \& Lemieux I (2006). Abdom obes \& metab synd. Nat 444(7121): 881-887.
4. Enas EA, Mohan V, Deepa M, Farooq S, Pazhoor S \& Chennikkara H (2007). The metabolic syndrome and dyslipidemia among Asian Indians: a population with high rates of diabetes and premature coronary artery disease. $J$ Cardiometab Syndr 2(4): 267-275.

5. Meshkani R \& Adeli K (2009). Hepatic insulin resistance, metabolic syndrome and cardiovascular disease. Clin Biochem 42(13-14): 1331-1346.

6. Soresi M, Noto D, Cefalu AB, Martini S, Vigna GB, Fonda M, Averna MR, (2013). Nonalcoholic fatty liver and metabolic syndrome in Italy: results from a multicentric study of the Italian Arteriosclerosis society. Acta Diabetol 50(2): 241-249.

7. Zimmet P, Alberti K \& Shaw J (2001). Global and societal implications of the diabetes epidemic. Nat 414(6865): 782787.

8. Lobo RA, (2008). Metabolic syndrome after menopause and the role of hormones. Matur 60(1): 10-18.

9. Carr MC, (2003). The emergence of the metabolic syndrome with menopause. $J$ Clin Endo \& Metabol 88(6): 2404-2411.

10. Wilson PW, D'Agostino RB, Parise H, Sullivan L, \& Meigs JB, (2005). Metabolic syndrome as a precursor of cardiovascular disease and type 2 diabetes mellitus. Circ 112(20): 30663072.

11. Malik S, Wong ND, Franklin SS, Kamath TV, L' Italien GJ, Pio JR, \& Williams GR, (2004). Impact of the metabolic syndrome on mortality from coronary heart disease, cardiovascular disease, and all causes in United States adults. Circ 110(10): 1245-1250.

12. Eshtiaghi R, Esteghamati A \& Nakhjavani M (2010). Menopause is an independent predictor of metabolic syndrome in Iranian women. Matur 65(3): 262-266. 
13. Gohlke-Bärwolf C (2000). Coronary artery disease-is menopause a risk factor Basic Res Cardiol 95(1): I77-I83.

14. Wang N, Kuang L, Han B, Li Q, Chen Y, Zhu C, Zhu C (2016). Folliclestimulating hormone associates with prediabetes and diabetes in postmenopausal women. Acta Diabetol 53(2): 227-236.

15. Grundy SM, Cleeman JI, Daniels SR, Donato KA, Eckel RH, Franklin BA \& Smith Jr SC (2005). Diagnosis and management of the metabolic syndrome: an American Heart Association/National Heart, Lung, and Blood Institute scientific statement. Circul 112(17): 2735-2752.

16. Jafar $\mathrm{TH}$, Qadri $\mathrm{Z}$ \& Chaturvedi $\mathrm{N}$ (2008). Coronary artery disease epidemic in Pakistan: more electrocardiographic evidence of ischaemia in women than in men. Heart 94(4): 408-413

17. Basit A, \& Shera AS (2008). Prevalence of metabolic syndrome in Pakistan. Metab Syndr Relat Disord 6(3): 171175.

18. Mohsin A, Zafar J, Nisar YB, Imran SM, Zaheer K, Khizar B \& Qazi, R. A. (2007). Frequency of the metabolic syndrome in adult type2 diabetics presenting to Pakistan Institute of Medical Sciences. J Pak Med Assoc 57(5): 235.

19. Ainy E, Mirmiran P, Asl SZ \& Azizi F (2007). Prevalence of metabolic syndrome during menopausal transition Tehranian women: Tehran Lipid and Glucose Study (TLGS). Matur 58(2): 150-155.

20. Ch Chowdhury, M, ZI, Anik A, M Farhana Z, Bristi PD, Al Mamun BA, Uddin MJ \& Rahman M (2018). Prevalence of metabolic syndrome in Bangladesh: a systematic review and meta-analysis of the studies. BMC Publ Heal 18(1): 308.

21. Pa Pandey S, Srinivas M, Agashe S, Joshi J, Galvankar P, Prakasam C \& Vaidya R (2010). Menopause and metabolic syndrome: A study of 498 urban women from western. India. $J$ Mid-Lif Healt 1(2): 63.

22. Fernandez ML \& Murillo AG (2016). Postmenopausal women have higher HDL and decreased incidence of low HDL than premenopausal women with metabolic syndrome. Paper presented at the Healthcare.

23. Chae CU, \& Derby CA (2011). The menopausal transition and cardiovascular risk. Obst \& Gyne Clin 38(3): 477-488.

24. Marchi RD, Dell'Agnolo CM, Lopes TCR, Gravena AAF, Demitt MDO, Brischiliari SCR \& Pelloso SM (2017). Prevalence of metabolic syndrome in pre-and postmenopausal women. Arch Endoc \& Metab 61(2): 160-166.

25. Mumusoglu S \& Yildiz BO (2019). Metabolic syndrome during menopause. Cur Vasc Pharm 17(6): 595-603. 